\title{
RELAÇÃO DAS OBRAS ENTRADAS NA BIBLIO- TÉCA DA FACULDADE DE DIREITO DE SÃO PAULO, NO PERIODO COMPREENDIDO ENTRE 16 DE MAIO E 15 DE AGOSTO DE 1935
}

\section{OBRAS GERAIS (0)}

Alfredo Issa Ássaly-Relatorio correspondente ao ano de $1934-\mathrm{Pe}-$ nitenciaria de São Paulo, São Paulo, 1935-Broch.-1 vol.-Doação.

Anales de la Universidad de ChileChile, 1934-Broch.-5 vols.-Permuta.

Annaes da Bibliotheca Nacional do Rio de Janeiro-Vol. XLIII, XLIV e XLV. Rio de Janeiro, 1931-Broch. 3 vols.-Permuta.

Annaes da Faculdade de Medicina da Universidade de São Paulo-Vol. X-São Paulo, 1934-Broch.-1 vol. -Permuta.

Annaire Interparlementaire-La vie politique et constitutionnelle des peuples-Paris, 1935-Encad.-1 vol. -Compra.

Annuario do Ensino do Estado de São Paulo-São Paulo, 1908-Broch. 1 vol.-Doação.

Annuario do Quinto Concilio Regional do Centro da Igreja Metodista do Brasil-São Paulo, 1935-Broch. -1 vol.-Doação.

Antonio de Padua Salles-Relatorio da Santa Casa de Misericordia de São Paulo em 1934-São Paulo, 1935 -Broch.-1 vol.-Doação.
Augusto Victorino Alves Sacramento Blake-Diccionario bibliographico brasileiro-Rio de Janeiro, 1883-1902 -Encad. 7 vols.-Compra.

A vida de Baurú-Baurú, 1933-Encad. 1 vol.-Doação.

A vida de Botucatú-Botucatú, 19321 vol.-Doação.

A vida de Itapetininga-Itapetininga, 1932-Encad.-1 vol.-Doaçäo.

A vida de Jaú-Jaú, 1933-Encad.-1 vol.

A vida de São Carlos-São Carlos, 1933 -Encad.-1 vol. Doação.

A vida de Sorocaba-Sorocaba, 1932Encad.-1 vol.-Doação.

Brazilio Machado-Relatorio apresentado ao Exmo. Sr. Dr. Rivadavia da Cunha Corrêa. Anno de 1911Rio de Janeiro, 1912-Broch.-1 vol. Doação.

Boletim do Instituto de EngenhariaVol. XXI-São Paulo, 1935-Broch.1 vol.-Permuta.

Boletim do Instituto da Ordem dos Advogados do Rio Grande do SulPorto Alegre, 1934-Broch.-1 vol.Permuta.

Boletim do Instituto Oscar FrieireSão Paulo, 1934-Broch.-1 vol.Permuta. 
Boletin de la Academia Argentina de Letras-Tomos I e II-Buenos Aires, 1933-1934-Broch._-2 vols.

Boletin de la Associacion Corredores de Seguros-Buenos Aires, 19291934-Broch.-1 vol.-Permuta.

Boletin de Ia Biblioteca del Congresso Nacional-Buenos Aires, 1934-1935Broch.-2 vols.-Permuta.

Boletin del Comite de Abogados de los Bancos de la Capital FederalBuenos Aires, 1934-Broch.-1 vol.

Boletin de Estatistica-Policia de la Capital-Annuario de 1934-Buenos Aires, 1935-Broch.-1 vol.

Boletin de Estatistica y Jurisprudencia-Policia de la Capital de la Republica Argentina-Buenos Aires, 1933-1934-Broch.-4 vols,-Permuta.

Boletin Mensual de Estatistica Agropecuaria-Republica Argentina, 1934 -Broch.-1 vol.-Permuta.

Candido de Figueiredo-Novo Dicionario da lingua portugueza-4." ed. -Lisboa, Encad.-2 vols.-Permuta.

Carlos Teschauer-Novo Dicionario Nacional-Porto Alegre, 1928-Encad. -1 vol.-Permuta.

Catalogos da Biblioteca "America"Santiago, 1927, 1929 e 1931-Broch.3 vols.-Permuta.

Catalogo da Biblioteca de Augusto Baillot-São Paulo, 1923-Broch.-1 vol. -Doação.

Chabure, A. de-A travers la PresseParis, 1914-Encad.-1 vol.-Doação.

Curso de Vacaciones para Extrangeiros -Universidad Internacional de Verano en Santander-España, 1935Broch.-1 vol.-Doação.

Diario da Justiça-Rio de Janeiro, 1934 -Encad.-1 vol.-Compra.

Diario Official do Estado de São Paulo -São Paulo, 1934-1935-Encad.—4 vol.-Doação.

Diario Official da Republica dos Estados Unidos do Brasil-Rio de Janeiro, 1934-1935-Encad. 4 vols.Compra.
Diario do Poder Legislativo-Rio de Janeiro, 1934-1935-Encad. 2 vols.Compra.

D. N. C.-Revista do Departamento Nacional do Café-Rio de Janeiro, Janeiro a Junho de 1935-Broch.-1 vol. -Permuta.

Dorvelino Guatemosim-Catalogo do Brasil de selos nacionaes postaes e telegraficos oficialmente emitidos -Rio de Janeiro, 1933-Broch.-1 vol.-Doação.

Dos años de labor en el Ministerio de Industrias-Republica Oriental del Uruguay, Montevideo, 1935-Broch.1 vol.-Permuta.

Egatea-Revista da Universidade Tecnica do Rio Grande do Sul-Porto Alegre, 1934-Broch.-1 vol.--Per muta.

El Presupuesto General de 1932 y el Estado presente de las finanzas nacionales de Hacienda de la NacionBuenos Aires, 1932-Broch.-1vol. -Permuta.

Encyclopaedia of the Social Sciences -Vol. XV-New Cork, 1935-Encad. -1 vol.-Compra.

F. de A. Carvalho Franco-Relatorio do Gabinete de Investigações-Sảo Paulo, 1935-Broch.-1 vol.-Doação.

Finanças dos Estados do Brasil-Rio de Janeiro, 1934-Broch.-3 vols.Doação.

Firmino Costa-Vocabulario analogico -São Paulo, 1933-Encad.-1 vol.Permuta.

Grandin, A.-Biobliographie générale des Sciences Juridiques, Politiques, Economiques et Sociales-Année 1934-Paris, 1935-Broch.-1 vol.Compra.

Idort-Orgão do Instituto de Organização Racional do Trabalho-São Paulo, 1934-Broch.-1 vol.-Permuta.

Inocencio Borges da Rosa-Nulidade do processo-Forto Alegre, 1935Broch.-1 vol.-Compra. 
J. T. da Silva Bastos-Dicionario Etimológico, Prosodico e Ortografico da lingua portuguêsa-Lisboa, 1928 -Encad.-1 vol.-Permuta.

Jornal do Commercio-Rio de Janeiro, 1934-1935-Encad.-4 vol.-Compra.

Journal des Économistes-Revue mensuelle de la Science économique et de la statistique-Paris, 1934-Tome CIV-Broch.-1 vol.-Compra.

La Revista Economica SudamericanaMontevideo, 1934-Broch.-1 vol.Permuta.

La Vie Intellectuelle-Tomo XXX, XXXV e XXXVI-1934-1935-Broch. -3 vols.-Permuta.

Le Mois-Synthese de l'activité mondiale-Paris, Février-Mars, Avril-Mai -1935-Broch.-2 vols.-Compra.

Memoria y Balance General-Ejercicio de 1934-Banco de la Nacion Argentina. Buenos Aires, 1935-Broch. -1 vol.-Permuta.

Murmurios Juvenis-Jornal Scientifico e Litterario-São Paulo, 1860-Encad. -1 vol.-Doação.

O Estado de São Paulo--São Paulo, 1934-1935-Encad.-4 vols.-Doação.

o Porvir-Jornal comercial, politico, literario, artistico e noticioso.-Rio de Janeiro, 1876-Encad.-1 vol.Doação.

Programme des cours du semestre d'été 1935-Université de Geneve, Geneve, 1935-Broch.-1 vol.-Permuta.

Relatorio do anno escolar de 1934, da Faculdade de Medicina de Porto Alegre.-Porto Alegre, 1934-Broch. -1 vol.-Doação.

Relatorio do Exercicio de 1934-Prefeitura Municipal de Piracicaba, Dezembro de 1934-Broch.-1 vol.Doação.

Revista do Archivo Municipal de São Paulo-São Paulo, 1934-1935-Broch. 10 vols.--Permuta.
Revista Brasileira-Rio de Janeiro, 1934-1935-Broch.-6 vols.-Doação.

Revista da Faculdade de Direito do Rio de Janeiro-Vols. IV e IX-Rio de Janeiro, 1908-1913-Broch._2 vols. -Doação.

Revista da Faculdade Livre de Direito do Estado do Pará-Pará, 1909,Broch.-1 vol.-Doação.

Revista de Identificacion y Ciencias Penales-Tomo IV-n. 17-La Plata, 1930-Broch.-1 vol.-Doação.

Revista do Instituto de Café do Estado de São Paulo-São Paulo, Janeiro a Junho de 1935-Broch,-1 vol. -Doação.

Revista Mexicana de Derecho Internacional-Tomo II-n. 1-Mexico, 1921 -Broch.-1 vol.-Doação.

Revista do Minho para o estudo das tradições populares-Barcelos, 1885 -Encad.-1 vol.-Doação.

Revista Nacional de Educação-Rio de Janeiro, 1932-1933-Broch.-II vols. -Permuta.

Revista Paulista de ContabilidadeSão Paulo, 1934-Broch.-1 vol.Doação.

Revista do Professor-Orgão do Centro do Professorado Paulista-São Paulo, 1934-1935-Broch.-1 vol.Permuta.

Revista da Universidade do Rio de Janeiro-Rio de Janeiro, Junho de 1934-Broch.-1 vol.-Permuta.

Revista Universitaria-Organo de la Universidad Menor del Cuzco. Cuzco, 1933, Broch.-1 vol.-Permuta.

Scarone (Arturo)-Apuntes para un Diccionario de Seudónimos y de publicacoines anónimas. Montevideo, 1934-Broch.-1 vol.-Permuta.

Sentinela da Monarchia-Orgão conservador-São Paulo, 1889-Encad.1 vol.-Doação.

The Association of the Bar of the City or New York-1934-Broch.-1 vol. -Permuta. 
Teller (F. X. de) Dictionnaire Historique, ou Histoire Abrégé. Paris, 18321833-Encad.-13 vols.-Permuta.

Valdez (João Fernandes)-Novissimo Diccionario Inglez-Portuguez e Portuguez-Inglez-Rio de Janeiro, Paris -Encad.-1 vol.-Compra.

Vicente Piragibe-Dicionario de Jurisprudencia Penal do Brasil-São Paulo, 1931-1934-Broch.-3 vols.Compra.

Viterbo (Souza) - A Livraria Real especialmente no Reinado de D. Manoel. Memoria apresentada á Academia Real das Sciencias de Lisboa.-Lisboa, 1901-Broch.-1 vol.Doação.

\section{PHILOSOPHIA (1)}

Bréhier, Émile-Histoire de la Philosophie-Paris, Broch. 4 vols.-Compra.

Dumas, Georges-Nouveau traité de Psychologie-Tome quatrieme- $\mathrm{Pa}$ ris, 1934-Encad.-1 vol-Compra.

Krishnamurti, J.-Collectanea de palestras-Rio de Janeiro-Broch.-1 vol.-Doação.

Krishnamurti, J.-O amigo immortal -Holland, Broch.-1 vol.-.

Krishnamurti, J.-Palestras e perguntas e respostas-Rio de Janeiro, 1934-Broch.-1 vol.-Doação.

Krishnamurti, J.-Relato verbal de palestras perguntas e respostas.Rio de Janeiro, 1934-Broch.-1 vol. -Doação.

Liscano, Tomas-La Moral del Abogado i de la Abogacia-Caracas, 1934 -Broch.-1 vol.-Doação.

Raul Briquet-Psicologia social-São Paulo, 1935-Broch.-1 vol.-Doação.

Silvio Vieiro Souto-e-Mario Barbosa Carneiro-Em torno do vóto supremo de Augusto Comte-Rio de Janeiro, 1928-Broch.-1 vol.-Doação.

Otavio Barboza Carneiro -Aguarda e conservação sociolátrica da Casa de Clotilde-Rio de Janeiro, 1930Broch.-1-vol.-Doação.

\section{RELIGIXO (2)}

Cerejeira, Manuel G.-A Igreja e o pensamento contemporaneo-Coimbra, 1930-Broch.-1 vol.-Compra.

Lopes, Francisco-Favores do Céo a Porftugal-Porto, 1871-Encad.-1 vol.-Doação.

Nunes, José Joaquim-Crónica da Ordem dos Frades Menores (1209. 1285)_Coimbra, 1918 -Encad.-1 vol. -Doação.

Pascal, Th.-Les sept principes de l'homme ou sa constitution occulte d'apers la théosophie-Paris, 1895Encad.-1 vol.-Doação.

\section{SOCIOLOGIA, DIREITO}

A. A. de Covello-A Lei de Segurança -São Paulo, 1935-Broch.-1 vol.Compra.

A. Alexandre Machado-O liberalismo economico e o proletariado-Maceib, 1935-Broch.-1 vol.-Doação.

Abgar Soriano de Oliveira-Da compra e venda com reserva de dominioRecife, 1934-Encad.-1 vol.-Compra.

Accordãos do Superior Tribunal de Justiça de Pernambuco-Recife, 19331934-Broch.-3 vols.-Permuta.

Aleixo Alves de Souza-Os grandes ideaes humanos-Rio de Janeiro, 1934-Broch.-1 vol.-Doação.

Aloysio de Carvalho Filho-Identificação pelos dentes-Rio de Janeiro, 1934 -Broch.-1 vol.-Doação.

Alvarez, Alejandro-La reconstrution du Droit des gens: Les données foudamentales et les grands principes duDroit International moderneBroch.-1 vol.-Doação.

Araujo Castro-A Nova Constituição Brasileíra-Rio de Janeiro, 1935Encad.-1 vol.-Compra.

Archivo Judiciario-Publicação quinzenal do "Jornal do Commercio"XXIII e XXXIV-Rio de Janeiro, 1935 -Broch.-2 vols.-Permuta. 
Archivos de Medicina Legal e Identificação-Anno V-n. ${ }^{\circ}$ 11-Rio de Janeiro, 1935-Broch.-1 vol.-Permuta.

Archivos de Philosophia do Direito e de Sociologia Juridica-n..$^{\circ}$ 1-2-Paris, 1935-Broch.1 vol--Compra.

4 Reorganização Financeira-Dois annos no Ministerio das Finanças -1928-1930-Coimbra, 1930-Borch. -1 vol..

Arminjon, P.-Précis de Droit International Privé-Paris, 1925-1931Encad.-3 vols.-Compra.

Arnoldo Medeiros da Fonseca.-Ação rescisoria por violação de direito exprésso-Rio de Janeiro, 1931Broch.-1 vol.-Doação.

Asembléa de Mutualidades EscolaresMadrid, 1934-Broch.-1 vol.-Permuta.

As Leis Sociaes em Vigor-Rio de Janeiro, 1934-Broch.-1 vol.-Compra.

Asúa, Luiz Jimenez de-Liberdade de amar e o direito a morrer-Lisboa, 1929-Broch.-1 vol.-Compra.

Athos A. de Magalhães-e-Getulio de PaulaSantos-Appellação n. ${ }^{\circ} 19.786$ de Santos-Broch.-1 vol.-Doação.

Bado, Augusto César-Discurso sobre aplicación de medidas extraordinarias $y$ censura a la prensa.-Uruguay, Mayo, 16 y 17 de 1935-Broch.-1 vol.-Permuta.

Benedicto Costa Netto-Honorarios de Agrimensor (divisão da Fazenda Araçatuba) - São Paulo, 1935-Broch. -1 vol.-Doação.

Beni Carvalho-Clovis Bevilaqua e o sentimento do direito-Ceará, 1935 -Broch.-1 vol.-Doação.

Bento de Faria-Pareceres-Rio de Janeiro, 1934-Broch.-1 vol.-Compra.

Beretta, Linita-La seconda celebrazione della "giornata della Madre e del Fanciullo"-Milano, 1935-Broch.1 vol.-Permuta.

Branco, Manoel Bernardes-Portugal e os estrangeiros-Lisboa, 1879Encad.-5 vols.-Doação.
Brites Ribas-Miranda de Vasconcelos e Alves Gomes-Lições de Historia do Direito Português-Coimbra, 1933

-Broch.-1 vol.-Compra.

Bruns, Viktor-L'égalité des droits de l'Allemagne en tant que probleme juridique-Berlin, 1935-Broch.-1 vol.-Permuta.

Bustamante, Antonio Sanchez de-Derecho Internacional Privado-Habana, 1931-Encad.3 vols.-Compra.

C. A. Saramdy Raposo-Theoria e pratica da cooperação-Rio de Janeiro, 1935-Broch.-1 vol.-Doação.

Caio Nelson de Senna-Apelação civi] n. ${ }^{\circ}$ 8.441, da Comarca de Santa Barbara-Bello Horizonte, 1935-Broch. -1 vol. Doação.

Capitant, Henri-Introduction a l'étude du Droit Civil-Paris, 1929-Broch. -1 vol.-Compra.

Capitant, M.-Droit Civil approfondé et comparé-Paris, 1932-1933-Encad. -1 vol.-Compra.

Cardozo de Mello Junior, Cardozo de Mello Neto e Luiz Nazareno F. de Assumpção. Apelação civil n. ${ }^{\circ}$ 13.991, de São Manoel-São Paulo, 1928-Broch.-1 vol.-Doação.

Carlos Ferraz Alvim-Dos direitos da mulher e sua evolução-Rio de Janeiro, 1934-Broch.-1 vol.-Doação.

Carlos da Silva Costa, Clovis Bevilaqua, J. X. Carvalho de Mendonça e Waldemar Ferreira-Renovação do registro de marca-Pareceres-São Paulo, 1929-Broch.-1 vol.-Doação.

Celso Spinola-Desapropriação por necessidade ou utilidade publica.Bahia, 1922-Broch.-1 vol.-Doação.

Celso Spinola-Embargos civeis de Itabuna-n. ${ }^{\circ} 360$-Bahia, 1928 -Broch.1 vol.-Doação.

Celso Spinola e Clovis Spinola-Agravo de petição da Capital-Bahia, 1933-Broch.-1 vol.-Doação.

Celso Spinola e Clovis Spinola-Embargos civeis de Ilhéos, n..$^{\circ} 1475$ Bahia, 1932-Broch.-1 vol.-Doação. 
Clovis Bevilaqua-Legitimate inheritance under the Brazilian Civil Law. -Iowa, 1935-Broch.-1 vol.-Doação.

Clovis Moreira Spinola-Agravo-A defeza dos direitos do Estado da Bahia, 1914-Broch.-1 vol.-Doaçăo.

Clovis Spinola-Recurso criminal da Capital-Bahia, 1934-Broch.-1 vol. -Doação.

Código do Notariado-Coimbra, 1932Broch.-1 vol.-Compra.

Code des Obligations de la Republique de Pologne-Paris, 1935-Broch.-1 vol.-Compra.

Código de Organizacion de los tribunales Civiles y de Hacienda - Edicion Oficial, 1934-Broch.-1 vol.-Permuta.

Código Penal--Montevideo, 1934Broch.-1 vol.-Permuta.

Código do Registro Civil-Coimbra, 1933-Borch.-1 vol.-Compra.

Coleção Geral da Legislação do Brazil -Rio de Janeiro, 1934-1935-Broch. -3 vols.-Doação.

Coll, Eduardo Albanel Mc-Legislacion sobre proxeentismo y delitos afines -Montevideo, 1932-Broch, -1 vol. Permuta.

Collecção das Leis e Decretos do Estado de São Paulo-Tomo XXXI e XLIV-São Paulo, 1931-1934-Broch. -2 vols..

Collecção das Leis da Republica dos Etsados Unidos do Brasil-Volume de 1933-Rio de Janeiro, 1934-Broch. -4 vols-Compra.

Collectaneas de Accordãos-Vol. VIBelo Horizonte, 1934-Broch.-1 vol. -Permuta.

Colombo Spinola-Acidentes no trabalho-0 valor de um globo ocular -Bahia, 1934-Broch.-1 vol.- Doação.

Commercio Exterior do Brasil-Directoria de Estatistica Economica e Financeira-Rio de Janeiro, 19301934-Broch.-1 vol.-Doação.
Congresso de Neurologia, Psiquiatria e Medicina Legal-Rio de Janeiro, 1932-Broch.-1 vol.-Permuta.

Conselho de Contribuintes-Vol. IV e V-Rio de Janeiro, 1935-Broch.2-vols.

Constituição do Estado de São Paulo de 9 de Julho de 1935-São Paulo, Broch-1 vol.-Doação.

Cooper, Rebecca-The Logical Influence of Hegel on Marx-Washington, 1925-Broch.-1 vol.-Doação.

Cuerpo Consular Extrangeiro-Buenos Aires, 1935-Broch.-1 vol.-Permuta.

Curti, Arthur-Manual de Derecho Mercantil Inglez-Madrid, 1931Ecand.-1 vol.-Compra.

Das Duplicatas ou Contas AsignadasCommissão de Constituição e Justica (Primeira Legislatura)-Camara dos Deputados, Rio de Janeiro, 1935 -Broch.-1 vol.-Permuta.

Decio Ferraz Alvim-Teoria da Instituição-Rio de Janeiro, 1934-Broch. -1 vol.-Doação.

Delos,J. J.-La Société Internationale et les principes du Droit PublicParis, 1929-Broch.-1 vol.-Compra.

Despachos De La Junta Consultiva Honoraria designada por decreto de P. E. de Octubre 18 de 1932-Buenos Aires, 1932-Broch.-1 vol.-Permuta.

Domingos Vellasco-Direito Eeleitoral -Rio de Janeiro, 1935-Broch.-1 vol.-Compra.

Dorvelino Guatemosin-Miscellanea Historica Postal e Philotelica Nacional-São Paulo, 1935-Encad.-1 vol.-Doação.

El Movimento del Estado Civil y la mortalidad de la Republica Oriental del Uruguay en el año 1933-Montevideo, 1934-Broch.-1 vol.-Doação.

En Plan de Acción Económica Nacional -Buenos Aires, 1934-Broch.-1 vol. -Permuta.

El Seguro de Maternidad-Madrid, 1934-Broch.-1 vol.-Permuta. 
Ennecerus, Ludwig-Tratado de Derecho Civil-Parte geral- Barcelona, 1934-1935-Broch.-2 vols.-Compra.

Estacio de Lima-A inversão dos sexos -Rio de Janeiro,-Broch.-1 vol.Compra.

Estatuto Judiciario-Coimbra, 1933Broch.-1 vol.-Compra.

Estatutos do Instituto de Pratica Profissional Juridica-São Paulo, 1935 -Broch.-1 vol.-Doação.

Estatutos, Regimento interno, Quadro social da Academia Brasileira de Letras-Rio de Janeiro, 1935-Broch. -1 vol.-Permuta.

Eurico Chaves, Antiogenes Chaves e Arlindo Figueiredo-Da verificação judicial de constas commerciaes para effeito de fallencia.-Recife, 1934Broch.-1 vol.-Doação.

F. Vergueiro Steidel-Apelação Civil n. 9.061, da Comarca de Bebedouro. -São Paulo, 1917-Broch.-1 vol. -Doação.

Fabio Luz Filho-Cooperativismo e credito agricola- São Paulo, s/d.Broch.-1 vol.-Compra.

Feroci, Virgilio-Diritto sindicale e corporativo-Milano, 1934-Broch.1 vol.-Compra.

Ferri, Henrique-Principios de direito criminal-O crimionso e o crimeSão Paulo, 1931-Broch.-1 vol.

Ferini, Contardo-Opere--Studi di Diritto Romano Bizantino-Milano, 1929-1930-Broch.-1 vol.-Compra.

Ficha Criminologica-Instituto de Criminologia d ela Republica Oriental del Uruguay-Broch.-1 vol.-Doação.

Foot, John Alderson-Private International Law-London, 1925-Encad.1 vol.-Compra.

Francisco Alexandre-Theoria e pratica do syndicalismo-Rio de Janeiro, 1955-Broch.-1 vol.-Compra.

Francisco Gonçalves Miranda-Memoria Historica da Imprensa Nacional -Rio de Janeiro, 1932-Broch.-1 vol.-Doação.
Francisco de Paula e Silva-Relatorio apresentado pelo Presidente da Côrte de Apelação-S. Paulo, 1935Broch.-1 vol.-Doação.

Frederico Pinedo, Gaston Léze y Luis Pierard-Los Proyectos Financieros ante la honorable Camaar de Deputados-(Discursos)-Buenos Aires, 1935-Broch.-1 vol.-Permuta. té-Tomepremier-Paris, 1935-Broc

Fuzier-Herman, Ed.-Code Civil annoté-Tome premier-Paris, 1935Broch.-1 vol.-Compra.

Gonçalves, Luiz da Cunha-Principios de Direito Corporativo-Lisboa, 1935 -Broch.-1 vol.-Doação.

Gonçalves, Luiz da Cunha-Tratado de Direito Civil em comentario ao Codigo Civil Português-Vol. VIICoimbra, 1934-Broch.-2 vols.Compra.

Gonzáles, Justo F.-Las actividades de la Comisión Nacional de Alimentacion Correcta-Ministerio de Instruccion Publica-Montevideo, 1933Broch.-1 vol.-Permuta.

Graziani, Augusto-Instituzioni di Scienza delle finanze-Torino, 1929, Encad.-1 vol.-Compra.

Gualtierotti, F. T. L.-Le ore decisive dll'Europe-Milano, 1935-Broch.-1 vol.-Compra.

Gudesten Pires-Ante-projecto da Lei de Sociedades Anonymas-Camara dos Deputados-Rio de Janeiro, 1935 -Broch.-1 vol.-Permuta.

Gudesten Pires-Sociedades Anonymas -Rio de Janeiro, 1935-Broch.-1 vol.-Compra.

Guia Fiscal-São Paulo, 1934-Broch. -1 vol.-Doação.

Heinsheimer, Karl-Derecho Mercantil -Barceolna, 1933-Encad.-1 vol.Compra.

Henrique Kalthoff-Da nacionalidade no direito internacional e na legislação comparada-São Paulo, 1935Broch.-1 vol.-Doação.

Hilario Freire-Nullidades processuaes da questão dos Pilões-São Paulo, 1911-Broch.-1 vol.-Doação. 
Horacio Berlinck-Seguro social do trabalhador (Conferencia) - São Paulo, 1935-Broch.-1 vol.-Doação.

Hueyo, Alberto-Discusion del proyecto e ley sobre conservacion de cédulas hipotecarias en el honorable Senado de la Nacion (Discurso)-Buenos Aires, 1932-Broch.-1 vol.-Permuta.

J. Pantoja Leite-A situação dos profesores das Escolas Superiores no Brasil-Rio de Janeiro, 1934-Broch. 1 vol.-Doação.

J. P. Porto Carrero-Psicologia Judiciaria-Rio de Janeiro, Broch.-1 vol.-Compra.

Jaffin, George H. - Prologue to Nomostatistics - New York, 1935Broch.-1 vol.-Doação.

Jean Servais etc E. Mechelinck-Les codes et les lois spéciales les plus uselles en vigoreur en BelgiqueBruxelles, 1935.-Encad. 1 vo.-Compra.

Jiménez, Inocencio-La unificación de los Seguros Socailes-Madrid, 1934 1 vol. Permuta.-(Broch.).

João Aristides Monteiro-Da natureza e da autonomia do Direito Internacional Privado--São Paulo, 1912Broch.-1 vol.-Doaçăo.

João Arruda-Ação de enriquecimento-São Paulo, 1919-Broch.-1 vol. -Doação.

João Arruda-Proletariado intellectual-Memorias apresentadas ao Conselho da Ordem-São Paulo, 1935Brochado.-1 vol.-Doação.

Joăo R. da C. Doria-Dos alcaloides, suas pesquizas medico-legaes. Bahia, 1933-Broch. 1 vol.-Doação.

João R. da C. Doria-Identificação ou reconhecimento de cadaver-Rio de Janeiro, 1935,-Broch.-1 vol.-Doação.

João R. da C. Doria-Perinecroscopia de corpo ou inspecção juridica-Rio de Janeiro, 1934-Broch. 1 vol.Doação.
João R. da C. Doria-Homicidios por envenenamento, alguns casos celebres. Rio de Janeiro, 1933-Broch.-1 vol. 一Doação.

Joăo R. da C. Doria-Estado da poeira do ar debaixo do ponto de vista higienico-Bahia, $\quad 1934$-Broch.-1 vol.-Doação.

Joâo R. da C. Doria-o homicidio na Bahia (Capital) no periodo de 1920 a 1933-Bahia, 1934-Broch.-1 vol. -Doação.

Joaquim Celso Moreira Spinola-Embargos civeis de Ilhéos-Bahia, 1919, Broch.-1 vol.-Doação.

José Candido Pimentel-Edificio de apartamentos-Estudo e comentarios sobre a propriedade de apartamento. Rio, 1925,-Broch. 1 vol.-Compra.

José Carlos de Matos Peixoto-Recurso extraordinario-_Rio de Janeiro, 1935 -Encad.-1 vol.-Compra.

Jurisprudencia-Coleccion Abadie-Santos-Montivideo, 1933-1934-Broch. 2 vols.-Permuta.

Justiça-Doutrina, Legislação, Jurisprudencia-Vol. V-Porto Alegre, 1934Broc.-1 vol.-Permuta.

Kuntz, Olive-Tiberius Caesar and the Roman Constituition-Washington, 1924-Broch. 1 vol.-Doação.

Los Proyectos Financeiros ante el honorable Senado-BuenoA Aires, 1935 -Broch.-1 vol.-Doação.

Laso, Enrique Sayagués-Suspencio condicional de la ejecucion de la pena. Montivideo, 1932-Broch.-1 vlo.-Permuta.

Laurenio Lago-Medalhas e condecorações brasileiras (Collectaneas de Actos Officiaes) 1808-1934-Rio de Janeiro, 1935-Broch. 1 vol.-Doação.

La Vie Juridique des Peuples-Paris, 1931-1934-Encad. 5 vols.-Compra.

L'E'conomie Dirigée-Conférencès organisées par la Société das éleves et auditeurs de l'E'cole Libre des Sciences Politiques-Paris, 1934Broch.-1 vol. 
Leite, João Pinto da Costa-Noçōes elementares de Economia PoliticaCoimbra, 1934-Broch. 1 vol. Compra.

Leyes, Decretos y Resoluciones de la Administración Vieira-Montivideo, 1919-Encad.-1 vol.-Permuta.

Ley $n .^{\circ}$ 11.582-Ministerio de Hacieda de la Nación-Buenos Aires, 19321 vol.-Permuta.

Ley Orgánica de la Universidad-Montevideo, 1934-Broch.-1 vol.-Permuta.

London \& Cambridge Economic Service -Monthly Bulletim-London, 1934Broch.-2 vols.-Compra.

Lopes Gonçalves-A Contsituição do Brasil-Rio de Janeiro, 1935-Broch. -1 vol.-Doação.

Lins e Silva-Discurso pronunciado na 1. ${ }^{a}$ sessão preparatoria da Assembléa Legislativa do Estado de Pernambuco, realizada em 11 de Julho 1935 -Recife, 1935-Broch.-1 vol.-Permuta.

Luiz Arthur Lopes e Alfredo Buchner Lopes da Cruz-O direito de renovação de Registro de Marcas de Fabrica e de Commercio-São Paulo, 1929 -Broch.-1 vol.-Doação.

Luiz Nazareno Teixeira de Assumpção -Da Sociedade-Do Juizo Arbitral. -São Paulo, 1934-Broch.-1 vol.Doação.

Manzini, Vincenzo-Trattado di Diritto Penale Italiano-Torino, 1933-1934Encad.-4 vols.-Compra.

Marx, Robert-La Justice pénale et les Personnes civilemant responsables du fait d'autre-Paris, 1935-Broch. -1 vol.-Compra.

Menano, Alberto-Economia Politica -Coimbra, 1927-Broch.-1 vol.Compra.

Menotti del Pichia, J. Assumpção Filho e Castro Andrade. A questão das Aguas Termaes de Lyndoia-São Paulo, 1920-Broch.-1 vol.-Doação.

Mensaje Explicativo de los Acontecimentos Revolucionarios-Montevideo, 1933-Broch.-1 vol.-Doação.
Merea, Manuel Pawlo-Resumo de Historia do Direito Português-Coimbra, 1925-Broch.-1 vol.-Compra.

Michaud, Léon-La Théorie de la personnalité morale et son application au droit Français-Paris, 1932 -Broch.-2 vols.-Compra.

Miguel Castello Branco, Celso Spinola e Antonio Salles Filho-Embargos civeis de Ilhéos, n. 751-Tribunal de Justipa da Bahia.-Bahia, 1925Broch.-1 vol.-Doação.

Miró, Amadeu Hurtado i-La Crisi del Dret-Barcelona, 1935-Broch.-1 vol. -Permuta.

Montalbano, Giuseppe-n Diritto Penale come Scienza-Palermo, 1935Broch.-1 vol.-Doação.

Montenegro, Arthur-A conquista do direito na sociedade romana-Coimbra, 1934-Broch.-1 vol.-Compra.

Mussolini, Benito-Quatro discursos relativos ao Estado CorporativoBroch.-1 vol.-Doação.

Nobajas, Armando Castro viejo y-Los principios christianos y la transformación y aun abolición del salariado (Discurso)-Santiago, 1921Broch.-1 vol.-Doação.

Noé Azevedo-Dos Tribunaes especiaes para delinquentes e como podem ser creados entre nós-S. Paulo, 1920Broch.-1 vol.-Doação.

Nuñez, Eduardo Rafael Nuñez yCodigo Civil-Habana, '1934-1935,Broch.-2 vols.-Compra.

Octavio Mendes-Recurso extraordinario-Alegações do recorrente-São Paulo, 1919-Broch.-1 vol.-Doação.

o Solo-Revista do Centro Agricola Luiz de Queiroz-Piracicaba, 1934 -Broch.-1 vol.-Doação.

Paulo Martins-Problemas Nacionaes -São Paulo, 1935-Broch.-1 vol.Doação.

Paulo Paulista de Ulhôa Cintra-Inquerito administrativo-Razões de defesa-São Paulo, 1934-Broch.-1 vol.-Doação. 
Pedro Baptista Martins-O abuso do direito e o acto illicito-Rio, 1935, -Broch.-1 vol.--Doação.

Piccola Enciclopedia Legale-L'Avvocato di tutti-Torino, 1931-Encad. -1 vol.-Compra.

Posada, Carlos G.-La conservacion de los direchos del emigrante en los seguros de invalidez, vejez y muerte ante la XVIII-Conferencia Internacional del Trabajo-Madrid, 1934-Broch.-1 vol.-Permuta.

Prestamos a Ayuntamientos para Finalidades Sociales-Madrid, 1935Broch.-1 vol.-Permuta.

Projecto para Estudo da Commissão de Finanças-Camara dos Deputados, Rio de Janeiro, 1934-Broch.-1 vol. -Permuta.

Projecto-Reorganiza o Tribunal de Contas e crea as suas Delegações. Camara dos Deputados, Rio de Janeiro, 1935-Broch.-1 vol.-Permuta.

Projecto n. 1-1935-Decreta o Codigo Penitenciario da Republica (1.a Legislatura) Camara dos Deputados, Rio de Janeiro, 1935-Broch.-1 vol. -Permuta.

Projecto n. 53-A-Prohibe as corridas de automoveis ou outros vehiculos, em aposta de velocidade; com parecer contrario da Comissão de Justiça-Camara dos Deputados, Rio de Janeiro, 1935-Broch.-1 vol. Doação.

Projecto n. 79-B-1935-Regula a execução do casamento religioso-Camara dos Deputados, Rio de Janeiro, 1935-Broch.-1 vol.-Doação.

Projecto n. 126-1935-Regula o casamento religioso para efeitos civis. Camara dos Deputados. Rio de Janeiro, 1935-Broch.-1 vol.-Permuta.

Projecto n. 741-1927-Decreta o Codigo Comercial Brasileiro-Camara dos Deputados, Rio de Janeiro, 1927Broch.-1 vol.--Permuta.

Rathenau, Walther-Critica de la época. Barcelona, Encad.-1 vol.-Compra.
Rathenau, Walther-Ou va le monde? -Paris, 1922-Encad.-1 vol.-- Compra.

Rébora, Juan Carlos-El Estado de Sitio y la ley histórica del desborde institucional-La Plata, 1935Broch.-1 vol.-Permuta.

Recueil Detudes sur les Sources du Droit-Paris, 1934 -Broch.-3 vols. -Compra.

Registro Nacional de Leyes, decretos y outros documentos-Montevideo, 1910-1928-Encad.-17 vols.Permuta.

Reglement de la Faculté de DroitUniversité de Geneve-Geneve, 1933 -Broch.-1 vol.-Permuta.

Regulamento da Faculdade de Direito de Goiaz-Estado de Goiaz, 1934Broch.-1 vol.-Permuta.

Reis, José Alberto dos-Curso de processo ordinário, sumário e sumarissimo civil e comercial-Coimbra, 1933-Broch.-1 vol.-Compra.

Revista da Côrte de Appellação-Vol. XI-Florianopolis, 1935-Broch.-1 vol.-Permuta.

Revista de Criminologia, Psiquiatria y Medicina Legal-Buenos Aires, 1934 -Broch.-1 vol.-Permuta.

Revista de Critica Judiciaria-Vol. XX e XXI-Rio de Janeiro, 1935-Broch. -2 vols.-Compra.

Revista do Fôro-Vols. XXIX e XXX -João Pessôa, 1933-1934-Broch.-2 vols.-Permuta.

Revista de Identificación y Ciencias Penales-La Plata, 1934-1935-Tomos $\mathrm{X}$ e XI-Broch.-2 vols.-Permuta.

Revista do Instituto Geographico e Historico da Bahia-n. 60-Bahia, 1934-Broch.-1 vol.-Permuta.

Revista Juridica de Catalunya-Vol. XL-Barcelona, 1934-Broch.-1 vol. -Permuta.

Revista dos Tribunaes-Vols. XVIII a XXIV e XXVI-Bahia, 1901 a 1932 e 1935-Broch.-3 vols.-Permuta. 
Revista dos Tribunaes-Vols. XCI e XCII-São Paulo, 1934-Broch.—2 vol.-Permuta.

Revue D'Économie Politique-Paris, 1934-Broch.-1 vol.-Compra.

Revue Trimestrielle de Droit CivilParis, 1934-Broch.-1 vol.-Compra.

Revue Trimestrielle de Droit CivilParis, 1934-Broch.-1 vol.-Compra.

Ricardo Gumbleton Daunt-Herschel e a Dáctyloscopia-São Paulo, 1934-1 vol.-Doação.

Revista del Diritto Commerciale-Milano, 1934 -Broch.-1 vol.-Compra.

Revista di Diritto InternazionaleRoma, 1934-Broch.-1 vol.-Compra.

Roberto de Ruggiero-Instituições de Direito Civil-S. Paulo, 1935-Broch. 1 vol.-Compra.

Rodrigo Ferraz Alvim-Da validade dos actos da Interventoria Daltro Filho -São Paulo, 1935-Broch.-1 vol.Doação.

Rodrigo Ferraz Alvim-Responsabilidade Civil do Estado-São Paulo, 1935-Broch.-1 vol.-Doação.

Rodrigo Octavio-Elementos de Direito Publico e Constitucional BrasileiroRio de Janeiro, 1935-Encad.-1 vol. -Doação.

Sardegna, Giuseppe Noto-Da "Realta" come limite della norma-Palermo, Broch.-1 vol.--Permuta

Sauer, Wilhelm-Filosofia juridica $\mathbf{y}$ social-Barcelona, 1933-Encard.-1 vol.-Compra

Seguro Obligatorio de MaternidadMadrid, 1934-Broch.-vol-Permuta

Spencer, Herbert-Facts and comments -Oxford, 1902-Encard.-1 vol.Doação

Studi Sassaresi-Vol. XII-Sassari, 1934-1935-Broch.-1 vol.-Permuta 1934-1935-Broch.-1 vol.-Permuta

Sylvio Romero Filho-A Codificação Americana do Direito Internacional Rio de Janeiro, 1927-Encad.-7 vols. -Doação
Tarde, G-Les lois de l'imitationParis, 1890-Encad.-1 vol.-Doação

Tarde, G-Les lois de l'imitationEtude sociologique-Paris, 1907Encad.-1 vol.-Doação

The Annals of the American Academy of Political and Social SciencePhiladelphia, May, July 1935-Broch. -2 vols.-Compra

Theophilo B. de Souza Carvalho-A Novação em Direito Romano e em Direito Civil-S. Paulo, 1914-Broch. -1 vol.-Doação

Torino, Enrique-Contratos a favor de terceiros-Buenos Aires, 1934-Broch. -1 vol.-Permuta

Valassi, Alvari-Direito Emphiteutico Coimbra, 1731-Encad.-1 vol-Doação.

Vasconcellos, José-Hispanoamérica frente a los nacionalismos agresivos de Europa y Norteamérica (Conferencias)-La Plata, 1934-1 vol.Permuta

Victor de Carvalho-Discurso-S. Paulo, 1934-Broch.-1 vol.-Doação

Vieira Ferreira-Consolidação das leis commerciaes de direito privado. São Paulo, 1935-Broch.-1 vol.-Compra.

Virgilio Marinho de Campos e Carlos Bana-Lei do divorcio-Lisboa, 1934 -Broch.-1 vol.-Compra.

Wagemann, Ernest-Estructura y ritmo de la economia mundial-Barcelona, 1933-Encad.-1 vol--Compra.

Waldemar Ferreira-Questão de Marca de Fabrica-São Paulo, 1935Broch.-1 vol.-Doação.

Weiss, André-La violation de la neutralité belge et luxembourgeoise par l'Allemagne-Paris, 1915-Broch.-1 -vol.-Doação.

\section{FILOLOGLA (4)}

Arthur Raggio Nobrega-Syntaxe do Infinito-Rio de Janeiro, 1930Broch.-1 vol.-Doação. 


\section{SCIENCLAS PURAS (5)}

Ameghino, Florentino-Obras comple$\operatorname{tar} y$ correspondencia cientificaVol. XVI-La Plata, 1934-Broch.-1 vol.-Permuta.

Boletim Astronomico e Geophysico do Serviço Astronomico e Meteorologico do Estado de São Paulo-São Paulo, 1928-Broch.-1 vol.-Doação.

Castelof, F. Jollivet-La Science Alchimique-Paris, 1904-Encad.-1 vol.Doação.

Renato Kehl-Como escolher um bom marido (Regras praticas)-2. ${ }^{\mathrm{a}}$ ed.Rio de Janeiro, 1935-Broch.-1 vol. -Doação.

Renato Kehl-Lições de Eugenia-Rio de Janeiro, 1935-Encad.-1 vol.Doação.

Revista da Exposição Antropologica Brasileira-Rio de Janeiro, 1882Encad.-1 vol.-Doação.

\section{SCIENCIAS APPLICADAS}

E. A. Graner-Contribuição para o estudo cytologico da mandioca-Piracicaba, 1935-Broch.-1 vol. Permuta.

James Ferraz Alvim-Centros de Malarioterapia e de prevenção de sifilis nervosa-São Paulo, 1935-Broch. -1 vol.-Doação.

James Ferraz Alvim-Dispensarios e serviços abertos para doentes mentaes. São Paulo, 1935-Broch.-1 vol. -Doação.

João R. da C. Doria-Estudo do ar confinado sob o ponto de vista higienico-Rio de Janeiro, 1934Broch.-1 vol.-Doação.

João R. da C. Doria-Da agua (estudo higienico) Bahia, 1934-Broch.-1 vol.-Doação.

Posada, Carlos-La solucion del proglema de las inundaciones y desagues en la Provincia de Buenos Aires-La Plata, 1934-Broch.-1 vol. -Permuta.
Quarta Conferencia Sulamericana de Higiene, Microbiologia e Patologia -Rio de Janeiro, 1931-Broch.-3 vols.-Permuta.

Theodoro Sampaio-O Estado da Bahia. Agricultura, criação de gado, industria e commercio-Bahia, 1925 -Broch.-1 vol.-Doação.

\section{BELAS ARTES (7)}

Ulisses Paranhos (Sintese de Historia da Arte) São Paulo, 1935-Broch.1 vol.-Doação.

\section{LITTERATURA (8)}

Analyse des Oubrages de J. J. Rousseau-Geneve-Paris, 1785-Encad.1 vol.-Doação.

Antici, Irene-La senda peralelaBuenos Aires, 1930-Broch.-1 vol. -Permuta.

Armando de Salles Oliveira-Discursos-São Paulo, 1935-Broch.-1 vol. -Doação.

Assis Cintra-A questão de limites entre São Paulo e Minas-São Paulo, 1935-Broch.-3 vols.-Compra.

Aureliano Leite-Discurso-Rio de Janeiro, 1935-Broch.-1 vol.-Doação.

Gaspar Correa-Lendas da IndiaLisboa, 1858-Encad.4 vols.-Doação.

José Perez-Uma interpretação do "Quixote" e as origens do Cinema no "Quixote" (Conferencias) Rio de Janeiro, 1935-Broch.-1 vol.Doação.

Leon, D. Salvador Cabeza-Discurso leido en la solemne inauguración del Curso Academico de 1917 a 1918 en la Universidad Literaria de Santiago-Broch.-1 vol.-Doação.

Mario Barbosa Carneiro-DiscursoRio de Janeiro, 1933-Broch.-1 vol. -Doação.

Marques da Cruz-Historia da Literatura-São Paulo-Rio de Janeiro,Encad.-1 vol.-Compra. 


\section{HISTORIA E GEOGRAFIA}

A Veneravel Ordem Terceira de São Francisco da Penitencia do Rio de Janeiro, desde a sua fundação até a commemoração do primeiro centenario do Brasil-Noticia Historica1822-1922-Broch.-1 vol.-Doação.

Alberto Rangel-Gastão de Orleans, o ultimo Conde d'Eu-São Paulo, 1935 -Broch.-1 vol.-Compra.

Alfredo Nascimento-O Centenario da Academia Nacional de Medicina do Rio de Janeiro-1829-1929-Rio de de Janeiro, 1929-Broch.-1 vol.Permuta.

Andler, Ch.-Le pan-germanismeParis, 1915-Broch,-1 vol.-Doação.

Anibal Mattos-Escritos e apontamentos sobre a vida de José de Anchieta. Bello Horizonte. 1934-Broch. -1 vol.-Compra.

Azevedo, Luiz Antonio de-Dissertação critico-filologico-historica-Lisboa, 1815-Encad.-1 vol.-Doaçäo.

Bédier, Joseph-Les crimes allemands d'apres des témoignages allemands. Paris, 1915-Broch.-1 vol.-Doação.

Candido Motta-São Paulo e a Republica-São Paulo, 1935-Broch.-1 vol.-Doação.

Carlos Xavier Paes Barreto-Feriados do Brasil-Rio de Janeiro, 1926Broch. 3 vols.-Permuta.

Cincoentenario da Estrada de Ferro do Paraná (1885-1935) Santa Catarina,-Broch.-1 vol.-Doação.

Clotilde de Vaux-Willelmine-Paris, 1929-Broch.-1 vol.-Doação.

Conferencia Celebrada na Academia Real das Ciências de Lisboa acerca dos descobrimentos e colonisações dos portuguêses na Africa-Lisboa, 1877-Encad.-1 vol.-Doação.

Durkhein, Emile-L'Allemagne au-dessus de tout-Paris, 1915-Broch.1 vol.-Doação.

Edgar Fontoura-Marcilio Dias-Rio de Janeiro-Broch.-1 vol.-Doação.
Emile Durkhein et E. Denis-Qui a voulu la guerre?-Paris, 1915-Broch. -1vol.-Doação.

E. Lavisse et $\mathrm{Ch}$. Audler-Pratique et dictrine allemands de la guerreParis, 1915-Broch.-1 vol.-Doação.

Eurico de Góes-Os Simbolismos Nacionaes-São Paulo, 1918-Encad.-1 vol.-Doação.

Felner, Rodrigo José de Lima-Nome verdadeiro do português João Fernandes Vieira, eelebre nas guerras de Pernambuco contra os Holandezes-Lisboa, 1875-Encad.-1 vol.Doaçã.o

Firmo, Mathias José de Oliveira dos Santos-Noticia sobre a vida de Pedro Alvares Cabral-Lisboa, 1875Encad.-1 vol.-Doação.

Frederico de Barros Brotero-Descendentes de José Fernando de Almeida Barros-São Paulo, 1934-Bruch.-1 vol.-Doação.

Historia da Colonização Portugueza do Brazil-Vol. III-fases. VII a XIIIRio de Janeiro-Broch. 1 vol.-Doação.

Kropotkin, Pedro $\rightarrow$ A grande revolução 1789-1793)-Rio de Janeiro, 19351 vol.-Doação.

Mac-Pherson, M. J. Gornes-Venezuela Grafica-Segundo Tomo-Caracas, 1930-Broch.-1 vol.-Doação.

Manuel Viotti-Veiga Filho (Patrono da cadeira XXVII) Esboço bio-bibliographico-S. Paulo, 1935-Broch. -1 vol.-Doação.

Mario de Lima Barbosa-Les Français dans l'Histoire du Brésil-Rio de Janeiro, Paris, 1923-Encad.-1 vol. 一Doaçăo.

Memoria del Instituto Nacional de Prevision en 1933-Madrid, 1934Broch.-1 vol.-Permuta.

Michel, R. Francisque-Paris, 1882Encad.-1 vol.-Doação.

Nina Rodrigues- $O$ animismo feitichista dos negros bahianos-Rio de Janeiro, 1935-Broch.-1 vol.-Compra. 
Noticias para a Historia e Geographia das Nações Ultramarinas-Lisboa, 1812-Encad.—7 vols.-Doação.

Obras Completas y correspondencia cientifica de Florentino AmeghinoLa Plata, 1913-1918-Broch.-15 vols. -Doação.

Odylio Costa Filho-Ensaio n. 1-Clovis Bevilaqua-Rio de Janeiro, 1935 -Broch.-1 vol.-Doação.

Pereira de Sá, Simão-Historia topografica e belica da Nova Colonia de Sacramento do Rio da Prata-Rio de Janeiro, 1900-Encad.-1 vol.Doação.

Rathenau, Walter-Le Kaiser-Paris, 1930-Broch.-1 vol-Compra.

Reiss, R. A.-Comet les Austro-Hongrois ont fait la guerre en Serbie -Paris, 1915-Broch.-1 vol.-Doação.

Revista do Instituto Archeologico, His. torico e Geographico Pernambucano -Vol. XXXII-Pernambuco, 1934Broch.-1 vol.-Permuta.

Revista do Instituto Geographico e Historico da Bahia-Vols. IX, LII e LVIII-Bahia, 1914-1932-Broch.-4 vols.-Permuta.

Revista do Instituto Historico e Geographico Brasileiro-Rio de Janei- ro, 1932-1933-Broch. -4 vols.-Permuta.

Revista do Instituto Historico e Geographico Parahybano-Vol. VIIIJoão Pesôa, 1935-Broch.-1 vol.Permuta.

Revista do Instituto Historico e GeoGraphico de São Paulo-Vol. XXX -São Paulo, 1935-Broch.-1 vol.Permuta.

Ricardo Severo-O Liceu de Artes e Oficios de São Paulo-Historico, estatutos, regulamentos, programas e diplomas, 1873-1934-Broch.-1 vol. -Doação.

Richet, Charles-Abrége d'histoire génerale-Paris, 1919-Encad.-1 vol. -Doação.

Segundo Congresso Pan-Americano de Tuberculose-Rio de Janeiro, 19301932-Broch.—3 vols.-Permuta.

Subsidios para a Historia da India Portuguêsa-Lisboa, 1868-Encad.1 vol.-Doação.

Viterbo, Souza-Trabalhos Nauticos dos Portuguêses nos seculos XVI e XVII-Memoria apresentada á Academia Real das Ciências-Encad.2 vols.-Doação. 